\title{
Numerical simulation of a 2-D jet-crossflow interaction related to film cooling applications: Effects of blowing rate, injection angle and free-stream turbulence
}

\author{
S SARKAR and T K BOSE \\ Department of Aerospace Engineering, Indian Institute of Technology, \\ Madras 600036, India
}

MS received on 3 October 1994

\begin{abstract}
The aerodynamics of a coolant jet in a hot crossflow in an environment representative of the gas turbine practice, is numerically investigated for film cooling applications. The time-dependent, massaveraged Navier-Stokes equations coupled with the compressible form of a two-equation low-Reynolds number $(k-\varepsilon)$ model are solved based on an explicit finite volume formulation. The computed flow-field and surface temperature distributions along with the turbulence quantities are presented to illustrate the variation of flow-physics and heat transfer phenomena which occur in a jet-crossflow interaction with changing blowing rates, slot angles, and inlet free-stream turbulence levels.
\end{abstract}

Keywords. Numerical simulation; slot-film cooling; turbulent flow.

\section{Introduction}

Components of a modern aircraft gas turbine are exposed to extremely high temperature. Control of both temperature level and gradient is needed for reliable operation and prolonged useful life of these components. Film cooling, one of the most popular methods, is widely used for this purpose. A cold secondary fluid is locally injected into the hot crossflow through a slot or a series of holes at one or more discrete locations along the surface to be protected. The secondary fluid is expected to create an insulating film which would reduce the convective heat transfer rate from the hot gas stream to the exposed surface. The flow-field near the injected coolant jet is very complex with high gradients of velocity, temperature, turbulence intensity and a local flow reversal (figure 1).

Studies on film cooling are widespread in the literature. Unfortunately, most of these investigations are only devoted to the analysis of surface heat transfer phenomena. Aerodynamic aspects related to the jet-mainstream interactions and the coolant layer formation are not yet completely understood. Numerical analysis, which provides detailed information of flow-field and heat transfer phenomena, can improve the 


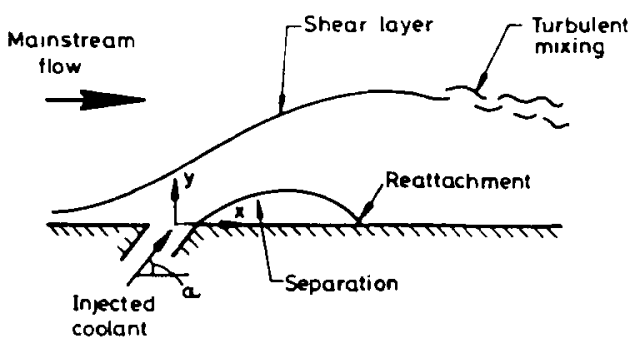

Figure 1. A general description of flow-field.

understanding of film cooling process when used in conjunction with experimental studies.

The highly complex flow-field created by the secondary fluid injection, depends on a wide variety of parameters such as the injection angle $(x)$, the blowing ratio $(m)$, the density ratio $(D R)$, the free stream turbulence intensity and the state of incoming boundary layer. An excellent review by Goldstein (1971) describes some of these factors and earlier experimental investigations on film cooling.

There are several experimental studies that measure the adiabatic wall temperature to define the film cooling effectiveness $(\eta)$ for different slot and hole injection configurations and also for different density and blowing ratios (Papell 1960; Petersen et al 1977; Foster \& Lampard 1980; Sinha et al 1990). The flow-field generated by injection from a row of holes is more complicated than that of slot injection because of threedimensionality. Aerodynamic investigations related to the film cooling rarely appear in the open literature. Among the very few, Andreopoulos \& Rodi (1984) and Pietrzyk et al (1989) made a detailed turbulence study and aerodynamic description of a jet in a crossflow.

The free-stream turbulence is a strong influencing parameter for the turbine blade cooling application. Experimental investigations (Brown \& Minty 1975; Ko \& Liu 1980 ) indicated a loss of cooling effectiveness with an increase of free-stream turbulence for both slot and hole injection due to greater mixing of the injected secondary air with the mainstream. Kadotani \& Goldstein (1979) reported the effects of free-stream turbulence, both in intensity and scale, on a turbulent jet. They concluded that the free-stream intensity and scale affected a number of parameters such as the boundary layer thickness, the height of the jet penetration and the mixing between the mainstream and the injected flow. Jumper et al (1991) pointed that a strong interaction with the cooling film would be expected as the free-stream turbulence levels are raised to a level comparable to that in the film. They concluded that the high turbulence levels of $14-17 \%$ could result in significant reduction in the film cooling effectiveness compared to the data bases with low turbulence level. Thus, the use of the data base with low free-stream turbulence would not be appropriate in assessing heat transfer phenomena for turbomachinery applications.

In the present study, the low-Reynolds number compressible form of a two-equation model, coupled with Favre-averaged mean flow equations (Favre 1965), is implemented into a purely explicit scheme which is applicable for a wide range of Mach numbers. There are limited attempts which incorporate the higher order turbulence models into an explicit solution, as it might have been thought that the stiffness associated with the 
explicit treatment is mainly due to the large source terms in the transport equations of turbulence models. But, the analysis of Kunz \& Lakshminarayana (1991) illustrates that the slower convergence rates that occur when explicit schemes are used to compute turbulent flows, are primarily due to the hyperbolic and parabolic stability constraints associated with the high grid clustering, and not due to the stiffness associated with large source terms in the transport equations of the turbulence models. Numerical results of flow and thermal fields along with turbulence quantities are presented to illustrate the effects of free-stream turbulence in a jet-crossflow interaction for film cooling applications in an environment representative of gas turbine practice. The variation of aerodynamics, shifting positions of flow reversal and change in film cooling effectiveness due to the change in blowing rate and injection angles are also discussed. The analysis is limited to a two-dimensional simulation.

\section{Governing equations and turbulence models}

In the present analysis, the density-weighted time-averaging (Favre 1965) is used. The density-weighted averaging leads to a simpler form of the governing equations with terms that are more amenable to physical interpretation. This is used for velocity components, temperature, turbulent kinetic energy and dissipation rate, whereas, the conventional time averaging is used for pressure, density, stress tensor and heat flux vector (Cebeci \& Smith 1974). The effects of fine scale turbulence are simulated by incorporating a two-equation model of turbulent kinetic energy $k$, and turbulent dissipation $\varepsilon$ and by specifying a turbulent Prandtl number $\operatorname{Pr}=0.9$. The low-Reynolds number terms of Lam-Bremhorst (1981) is used here in order to improve near wall modelling.

The two-dimensional, time-dependent, mass-averaged compressible Navier-Stokes equations alongwith two additional equations for $k$ and $\varepsilon$ can be expressed for each discrete quadrilateral cell in the integral form as,

$$
\frac{\partial}{\partial t} \int_{\Omega} \mathbf{U d} \Omega+\int_{S} \overline{\mathbf{Q}} \cdot \mathrm{d} \overline{\boldsymbol{S}}=\int_{\Omega} \mathrm{Hd} \Omega,
$$

where

$$
\begin{aligned}
\mathbf{U} & =\left[\begin{array}{l}
\rho \\
\rho u \\
\rho v \\
\rho e \\
\rho k \\
\rho \varepsilon
\end{array}\right] ; \quad \overline{\mathbf{Q}}, \text { the flux vector }=(\mathbf{F}, \mathbf{G}) \\
\mathbf{F} & =\left[\begin{array}{l}
\rho u \\
\rho u^{2}+p-\tau_{x x} \\
\rho u v-\tau_{x y} \\
\left(\rho e+p-\tau_{x x}\right) u-\tau_{x y} v+q_{x} \\
\rho u k-\left(\mu+\mu_{t} / \sigma_{k}\right) \partial k / \partial x \\
\rho u \varepsilon-\left(\mu+\mu_{t} / \sigma_{\varepsilon}\right) \partial \varepsilon / \partial x
\end{array}\right]
\end{aligned}
$$




$$
\begin{aligned}
& \mathbf{G}=\left[\begin{array}{l}
\rho v \\
\rho u v-\tau_{y x} \\
\rho v^{2}+p-\tau_{y y} \\
\left(\rho e+p-\tau_{y y}\right) v-\tau_{y x} u+q_{y} \\
\rho v k-\left(\mu+\mu_{t} / \sigma_{k}\right) \partial k / \partial y \\
\rho v \varepsilon-\left(\mu+\mu_{t} / \sigma_{\varepsilon}\right) \partial \varepsilon / \partial y
\end{array}\right] \\
& \mathbf{H}=\left[\begin{array}{l}
0 \\
0 \\
0 \\
0 \\
P_{k}-\rho \varepsilon \\
c_{\varepsilon 1} f_{\varepsilon 1} P_{k} \varepsilon / k-c_{\varepsilon 2} f_{\varepsilon 2} \frac{\rho \varepsilon^{2}}{k}
\end{array}\right]
\end{aligned}
$$

The total stress tensor and the heat flux vector are given in Cartesian coordinates as:

and

$$
\tau_{i j}=\mu\left[\left(\frac{\partial u_{i}}{\partial x_{j}}+\frac{\partial u_{j}}{\partial x_{i}}\right)-\frac{2}{3} \delta_{i j} \frac{\partial u_{k}}{\partial x_{k}}\right]+\tau_{t i j},
$$

$$
q_{i}=-\frac{\mu c_{p}}{\operatorname{Pr}} \frac{\partial T}{\partial x_{i}}+q_{t}
$$

In the present study, the laminar viscosity coefficient $\mu$ is assumed to be a function of temperature only, and is evaluated following Sutherland's law. The perfect gas equation of state $p=\rho R T$, is also considered to be applicable where,

$$
T=\frac{1}{c_{v}}\left[e-\frac{V^{2}}{2}\right]
$$

In preceding expressions, $\tau_{t i}$ and $q_{t_{1}}$ represent the turbulent stress tensor and heat flux vector, which are to be evaluated for the closure of (1). Incorporating an eddy viscosity formulation, the turbulence stress tensor and heat flux vector are given by:

and

$$
\tau_{t i j}=\mu_{i}\left[\left(\frac{\partial u_{i}}{\partial x_{j}}+\frac{\partial u_{j}}{\partial x_{i}}\right)-\frac{2}{3} \delta_{i j} \frac{\partial u_{k}}{\partial x_{k}}\right]-\frac{2}{3} \delta_{i j} \rho k
$$

$$
q_{t}=-\left(\frac{\mu_{t} c_{p}}{\mathrm{Pr}_{t}}\right) \frac{\partial T}{\partial x_{i}}
$$

where, the eddy viscosity

$$
\mu_{t}=c_{\mu} f_{\mu}\left(\rho k^{2} / \varepsilon\right)
$$

The production term $\boldsymbol{P}_{k}$ is expressed in Cartesian coordinates as:

$$
P_{k}=\tau_{t_{x x}} \frac{\partial u}{\partial x}+\tau_{t_{x x}}\left(\frac{\partial u}{\partial y}+\frac{\partial v}{\partial x}\right)+\tau_{t_{y}} \frac{\partial v}{\partial y},
$$

Here, $c_{\mu}, c_{\varepsilon 1}, c_{\varepsilon 2}, \sigma_{k}$ and $\sigma_{\varepsilon}$ are the model constants. $f_{\mu}, f_{\varepsilon 1}, f_{\varepsilon 2}$ are the damping functions used to simulate the low-Reynolds number effects. For the Lam-Bremhorst 
$(k-\varepsilon)$ model, considered in the present analysis, they are as follows:

$$
\begin{aligned}
& f_{\mu}=\left[1-\exp \left(-0.0165 \operatorname{Re}_{y}\right)\right]^{2}\left(1+20 \cdot 5 / \operatorname{Re}_{T}\right) \\
& f_{\varepsilon 1}=1+\left(0.05 / f_{\mu}\right)^{3} \\
& f_{\varepsilon 2}=1-\exp \left(\operatorname{Re}_{T}^{2}\right) \\
& c_{\mu}=0.09 ; c_{\varepsilon 1}=1.44 ; c_{\varepsilon 2}=1.92 ; \sigma_{k}=1.0 \text { and } \sigma_{\varepsilon}=1.3 .
\end{aligned}
$$

On solid wall, $k=0$ and $\varepsilon=(\mu / \rho)\left(\partial^{2} k / \partial y^{2}\right)$.

It should be noted that, although the $k-\varepsilon$ equations have been cast in compressible form, the modelling assumptions invoked here are essentially those for incompressible flow. The terms containing density fluctuation and pressure diffusion are neglected.

\section{Numerical procedure}

The unsteady equations (1) are marched in time using the explicit, second order accurate time-split, cell centred finite volume scheme based on MacCormack \& Paullay (1972) until the steady state is achieved. The advantage of a time-split system is that the computation proceeds with larger time increments as the stability criteria are less stringent.

In order to maintain stability in the presence of steep gradient of flow variables and to suppress any odd-even decoupling in the numerical solution, artificial dissipation terms are added to the finite volume formulation. Artificial dissipation used here is similar to that derived by Jameson et al (1981), which is a blend of second and fourth order differences with coefficients that depend on local pressure gradients. Thus, the artificial dissipation is introduced in the present formulation by defining an effective numerical flux across the cell face as,

$$
\mathbf{F}_{i+1 / 2, j}^{(A V)}=\mathbf{F}_{i+1 / 2, j}-\mathbf{D}_{i+1 / 2, j}
$$

where, $\mathbf{F}_{i+1 / 2, j}$ is the physical flux and $\mathbf{D}_{i+1 / 2, j}$ is the dissipation term, given by

$$
\begin{aligned}
\mathbf{D}_{i+1 / 2, j}=\sigma_{i+1 / 2, j}\left[\varepsilon_{i+1 / 2, j}^{(2)}\right. & \left(\mathbf{U}_{i+1, j}-\mathbf{U}_{i, j}\right) \\
& \left.-\varepsilon_{i+1 / 2, j}^{(4)}\left(\mathbf{U}_{i+2, j}-3 \cdot \mathbf{U}_{i+1, j}+3 \cdot \mathbf{U}_{i, j}-\mathbf{U}_{i-1, j}\right)\right] .
\end{aligned}
$$

The scaling factor

$$
\sigma_{i+1 / 2 . j}=\max \left(\Omega_{i+1, j} / \Delta t, \Omega_{i, j} / \Delta t\right)
$$

The coefficients $\varepsilon^{(2)}$ and $\varepsilon^{(4)}$ use the pressure as a sensor for shocks and stagnation points, and they are defined as

$$
\begin{aligned}
\varepsilon_{i+1 / 2, j}^{(2)} & =k^{(2)} \min \left[\max \left(v_{i+1 . j}, v_{i, j}\right), 0 \cdot 5\right] \cdot \min \left(M_{i+1 / 2, j} / M_{\alpha .} 1\right), \\
\varepsilon_{i+1 / 2 . j}^{(4)} & =\max \left[0,\left(k^{(4)} \min \left(M_{i+1 / 2, j} / M_{x}, 1\right)-\varepsilon_{i+1 / 2, j}^{(2)}\right)\right], \\
v_{i, j} & =\frac{\left|p_{i+1, j}-2 p_{i, j}+p_{i-1, j}\right|}{\left|p_{i+1, j}\right|+2\left|p_{i, j}\right|+\left|p_{i-1, j}\right|},
\end{aligned}
$$

where $k^{(2)}$ and $k^{(4)}$ are constants set at $1 / 4 \& 1 / 256$ respectively. To reduce the effect of artificial dissipation within the viscous region of flow, a function of local Mach number normalised with respect to the free-stream Mach number, the maximum value of which is limited to unity, is used here. Similar dissipation terms are defined in the $j$-direction. 


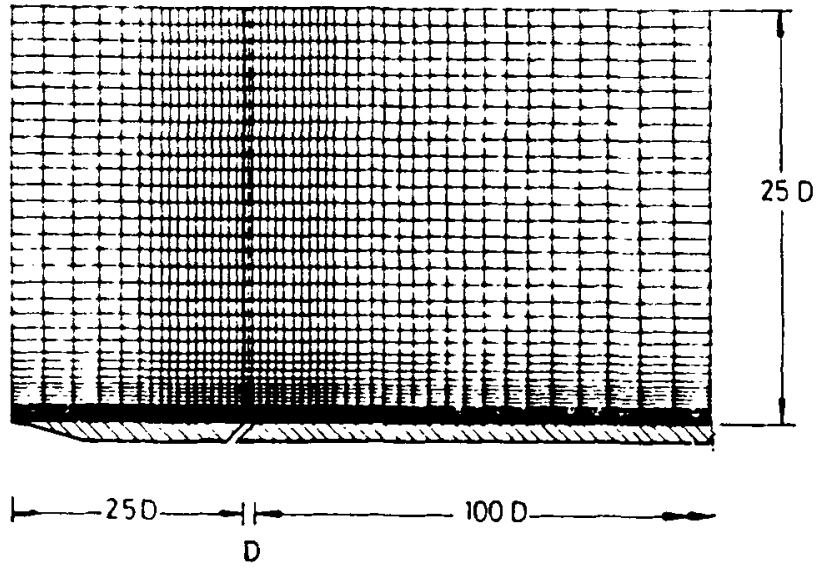

Figure 2. Computational grid.

\section{Grid generation}

The geometry consists of a flat plate with a slot of width $D=6.35 \mathrm{~mm}$, represented in figure 2. An algebraically generated non-uniform Cartesian computational mesh, consisting of $60 \times 50$ grid points in $x$ and $y$ directions respectively, is used. For computational purposes, the geometry is divided into the following three regions: (i) the region near the slot where most of the interaction takes place;(ii) the region between the inlet and the beginning of the interaction zone; and (iii) the rest of the computational domain. The grid in the $x$-direction is generated judiciously by placing a number of fine uniform grid points in the interaction region, within the slot and stretching the grid towards the inlet and the exit. A fine exponentially stretched mesh spacing is employed in the region near the wall for resolving viscous layer and a course uniform mesh spacing is used in the outer region. The first mesh point above the plate surface is chosen such that the average $y^{+}$is of the order of unity.

\section{Boundary conditions}

At the inlet, the total pressure, total temperature and the flow angle are specified; the static pressure is extrapolated from interior and hence the other variables at the inlet are updated for each time integration. The exit plane is positioned considerably far downstream of the injection $(100 \mathrm{D})$ so that a zero gradient condition is applied for all variables at this plane. On solid surfaces, no-slip conditions are imposed along with vanishing normal temperature gradient to simulate the adiabatic wall condition. The upper boundary of the computational domain is chosen sufficiently far from the plate and a 'slip-wall' type boundary condition seems to be appropriate. Thus, a slip condition is imposed by setting $v$-component of velocity and normal gradients of all other dependent variables to zero. The two-equation model needs specification of $k$ and $\varepsilon$ values at inlet. The constant values of $k$ and $\varepsilon$ are imposed at the inflow boundary based on the specified free-stream turbulence intensity and length scale as,

$$
\begin{aligned}
& k_{\alpha}=1.5 T u_{\alpha}^{2} V_{\alpha}^{2}, \\
& \varepsilon_{\alpha}=c_{\mu}^{3 / 4} k^{3 / 2} / l_{\alpha} .
\end{aligned}
$$


The free-stream turbulence intensity is considered as $2-12 \%$.

The experimental study of Pietrzyk et al (1989) indicates that a uniform mean velocity profile at the jet exit may be a better approximation of the actual film cooling conditions. Therefore, across the slot exit the following uniform conditions are applied.

$$
\begin{aligned}
& \rho u=m_{s} \cos x, \\
& \rho r=m_{s} \sin x, \\
& T=T_{s}, \\
& p=p_{s}, \\
& k=1.5 T u_{s}^{2} V_{s}^{2}, \\
& \varepsilon=c_{\mu}^{3 / 4} k_{s}^{3 / 2} / 0.5 \mathrm{D},
\end{aligned}
$$

where $T u_{s}$ is considered as $8 \%$ here.

\section{Results and discussions}

\subsection{Model validation}

Comparisons of the computed flow-field with experiment are presented in this section. All computations have been made on a PC-AT 486 based machine which has a CPU speed of 1 MFLOPS. The solutions are assumed to be converged when the logarithmic value of residuals have dropped by at least four orders of magnitude. Steady state has been achieved in 25,000 to 30,000 time steps.

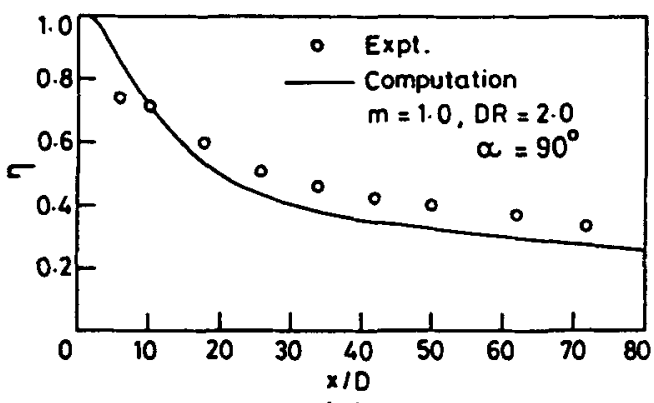

(a)

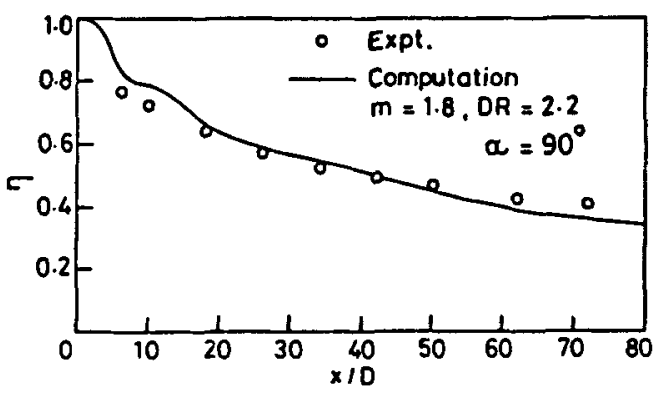

(b)
Figure 3. Streamwise variation of adiabatic film cooling effectiveness for two different blowing and density ratios. Experimental values from Papell (1960). 
A majority of experiments, appearing in the open literature on film cooling for the gas turbine practice and consequent computational studies made to simulate those experiments, have considered low free-stream velocity. An exception is the work of Papell (1960) where the free-stream conditions were more representative of the gas turbine environment with $\mathrm{M}_{x}=0.5$ or above. $T_{x}=790 \mathrm{~K}$ and $D R \simeq 2.0$. For this reason, the data set provided in Papell (1960) is chosen for validation.

Figures $3 \mathrm{a}$ and $\mathrm{b}$ compare the predicted and measured film cooling effectiveness downstream of a normally injected coolant jet for different blowing ratios, $m=1.0$ and 1.8 with $D R=2 \cdot 0$. Although the overall streamwise trend is correct, the film cooling effectiveness is under-predicted to some extent at $m=1.0$ (figure 3a). At a relatively higher blowing ratio of $m=1.8$ (figure $3 \mathrm{~b}$ ). predicted distribution of film cooling

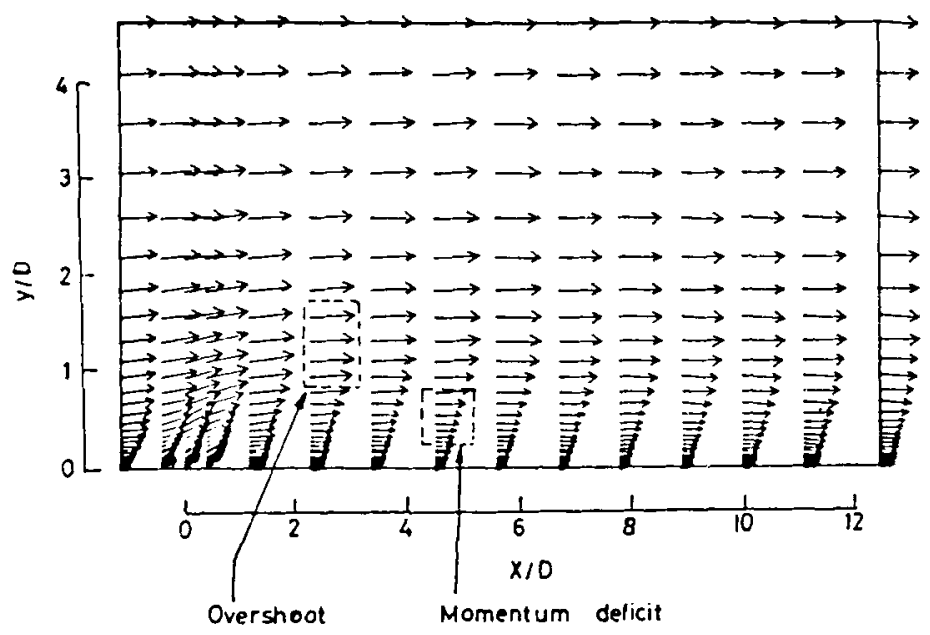

(a)

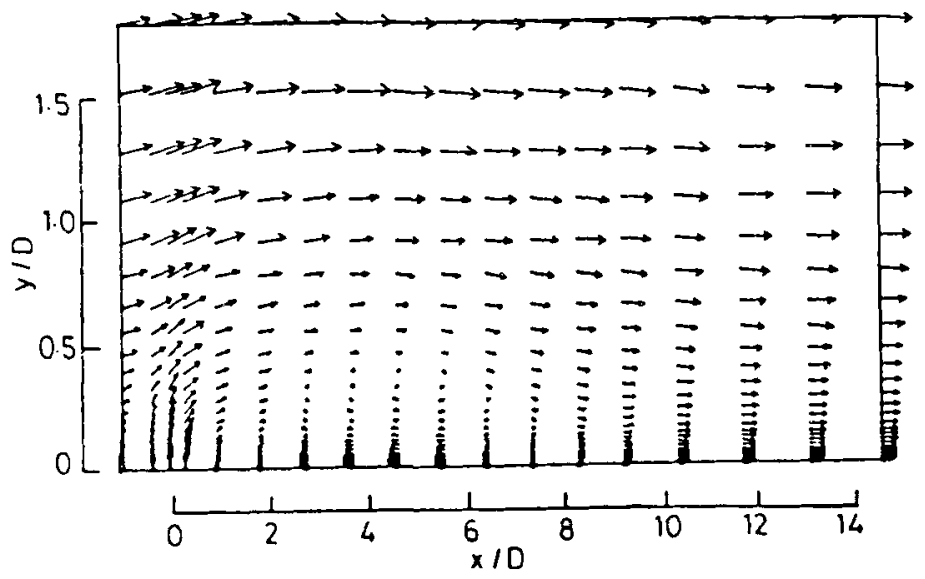

(b)

Figure 4. Computed velocity field (a) $m=1 \cdot 0, \alpha=45 ;$ (b) $m=1 \cdot 0, \alpha=90^{\circ}$. 

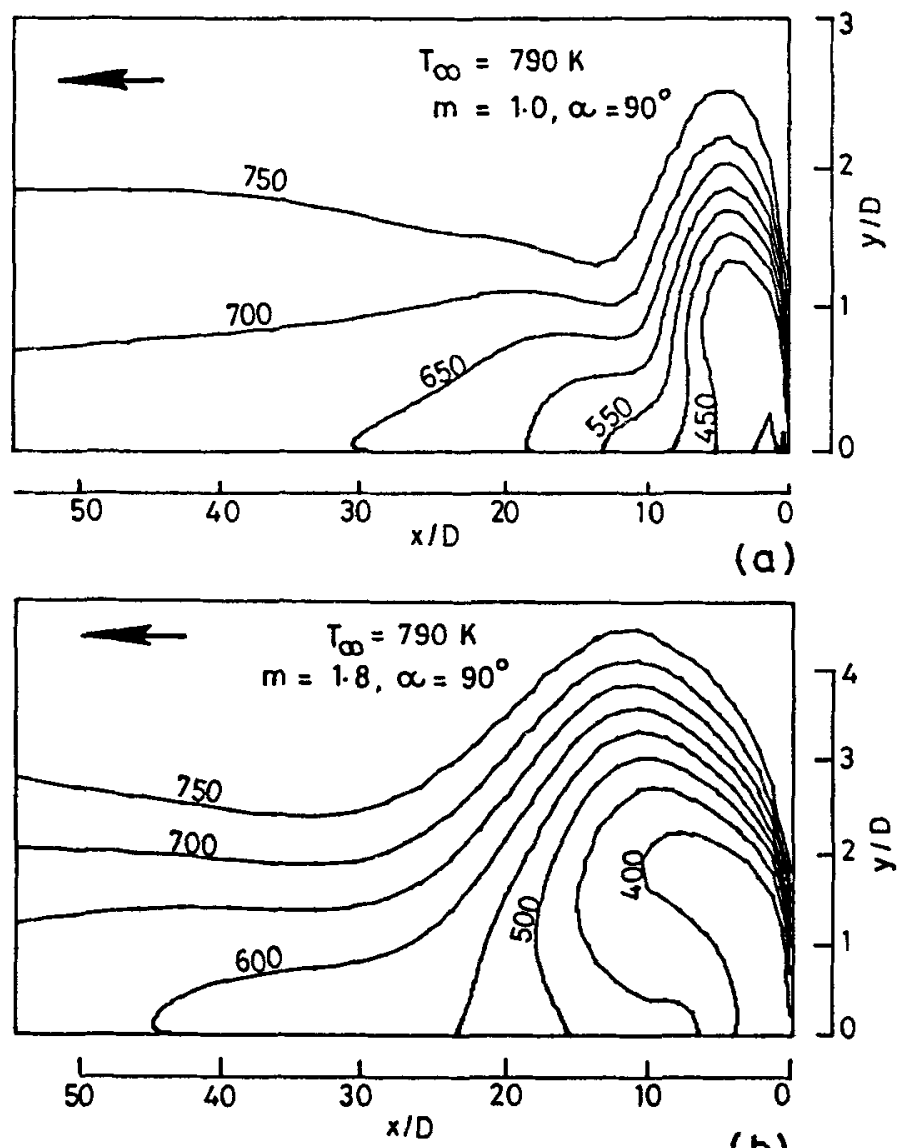

(b)

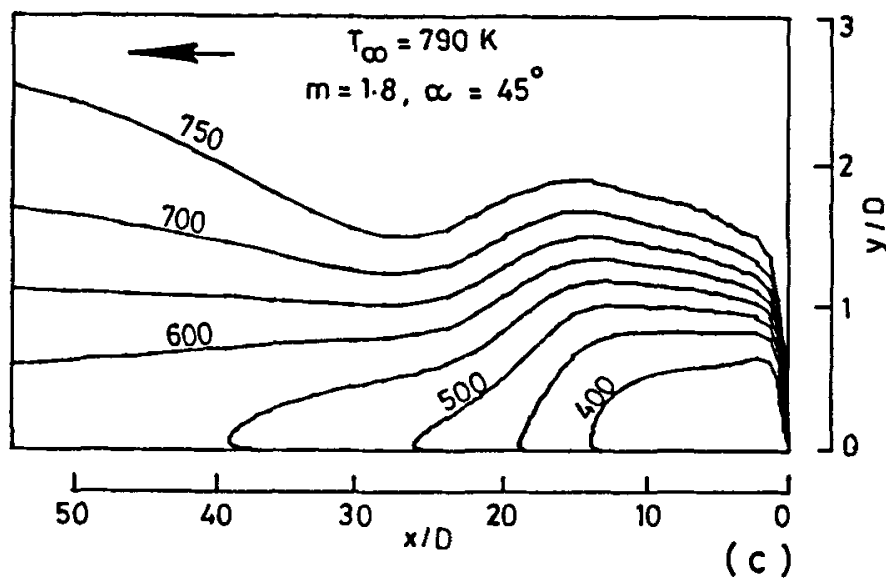

Figure 5. Computed temperature contours indicating effect of blowing rate and injection angle on the coolant jet trajectory due to different blowing ratios and injection angles. 
effectiveness matches very well with experiment. The under-prediction of film cooling effectiveness for a relatively lower blowing rate may possibly be attributed to the over-predicted mixing by the turbulent model used in the present study. Another uncertainty is that the upstream boundary layer thickness and its character is not clearly specified in Papell (1960). Hence, for the present study, the length upstream of the slot is fixed as $x / D=25$, considering the fact that the influence of upstream boundary layer is not very strong for slot injection (Seban 1960), though this may slightly affect the prediction. The free-stream turbulence considered here is $2 \%$ which gives the turbulent velocity profile near the leading edge. However, the overall magnitude and trend of effectiveness are reasonably well predicted by the present flow-solver. On this basis, the variation of flow-field and heat transfer phenomena with changing blowing rate, injection angle and free-stream turbulence are numerically investigated.

\subsection{Numerical results}

6.2a Effects of injection angles and blowing rates: The computed velocity field downstream of an injected jet at an angle of $45^{\circ}$ for $m=1.0$ and $D R=2$ is shown in figure $4 \mathrm{a}$. These velocity profiles are very similar to those measured by Pietrzyk et al (1989) and they clearly demonstrate a near-field wake region and a far-field relaxation region. In the wake region, the velocity profile exhibits two distinguishable zones, (i) the momentum deficit zone indicating the development of a shear layer between the outer edge of the injected jet and the crossflow, and (ii) right above the momentum deficit

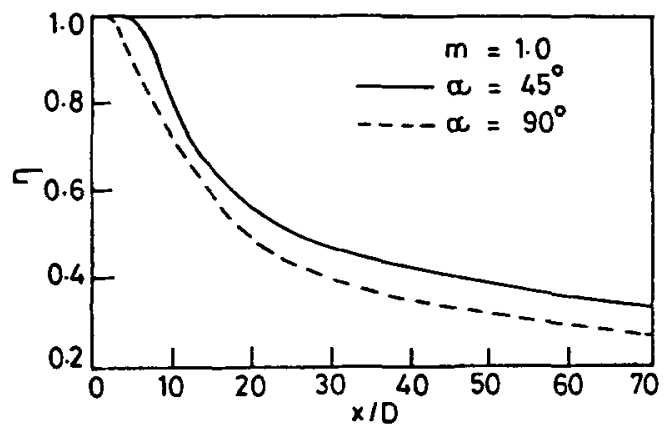

(a)

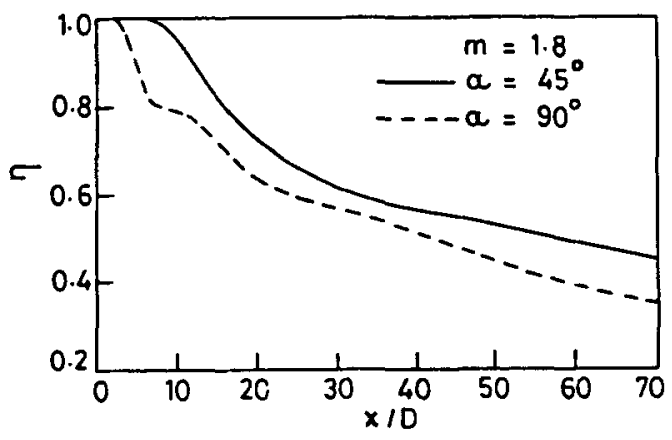

(b)
Figure 6. Computed streamwise variation of adiabatic film cooling effectiveness demonstrating effects of blowing rate $m$ and injection angle. 
zone, a region with a slight velocity overshoot caused by the crossflow accelerating over the coolant jet. There is no flow separation downstream of the slot exit. For the same blowing rate, as $\alpha$ is increased to $90^{\circ}$, the aerodynamics of the jet in crossflow has remarkably changed (figure $4 \mathrm{~b}$ ). The jet penetrates more into the mainstream, bends over, reattaches to the surface forming a separation bubble and subsequently flows downstream approaching the boundary layer type flows. Injected coolant undergoes turbulent mixing with the mainstream while crossing over the bubble and at the same time, a small fraction of the injected coolant mass drifts into the recirculating bubble, inducing cooling action near the slot. This leads to high effectiveness very near the slot exit with downstream flow separation also for 2-D slot injection. But in 3-D hole injection, the region beneath the jet is ventilated by the mainstream and a low effectiveness occurs.

One important aspect for film cooling processes is the jet trajectory which depends mainly on the injection angle and the momentum ratio. A high trajectory implies deeper penetration of jet into the crossflow, development of a reversal zone, pronounced flow mixing and hence less effectiveness. On the other hand, a low trajectory results in an attached coolant layer, less mixing and high effectiveness. In the present study, temperature field is used to determine the trajectory of the coolant jet.

Figures $5 \mathrm{a}$ and $\mathrm{b}$ illustrate the temperature contours for jet-crossflow interaction with $D R \simeq 2$ and $m=1.0$ and 1.8 respectively, for $\alpha=90^{\circ}$. As seen from the above figures, with the increase of blowing ratio, the coolant tends to retain its wall-jet character with strong penetration into the crossflow and thereby makes a high trajectory. To show the influence of injection angles on the trajectory, the temperature contours due to $\alpha=45^{\circ}$ for $m=1.8$ are presented in figure 5c. This clearly demonstrates that for the low angle of injection, the coolant hugs the surface to be protected, retaining its identity for a larger length. Thus, under this condition, high cooling effectiveness is expected even far-field. As the angle of injection increases, the coolant penetrates more into the crossflow resulting in more jet dilution which would cause a rapid decay of film-cooling effectiveness.

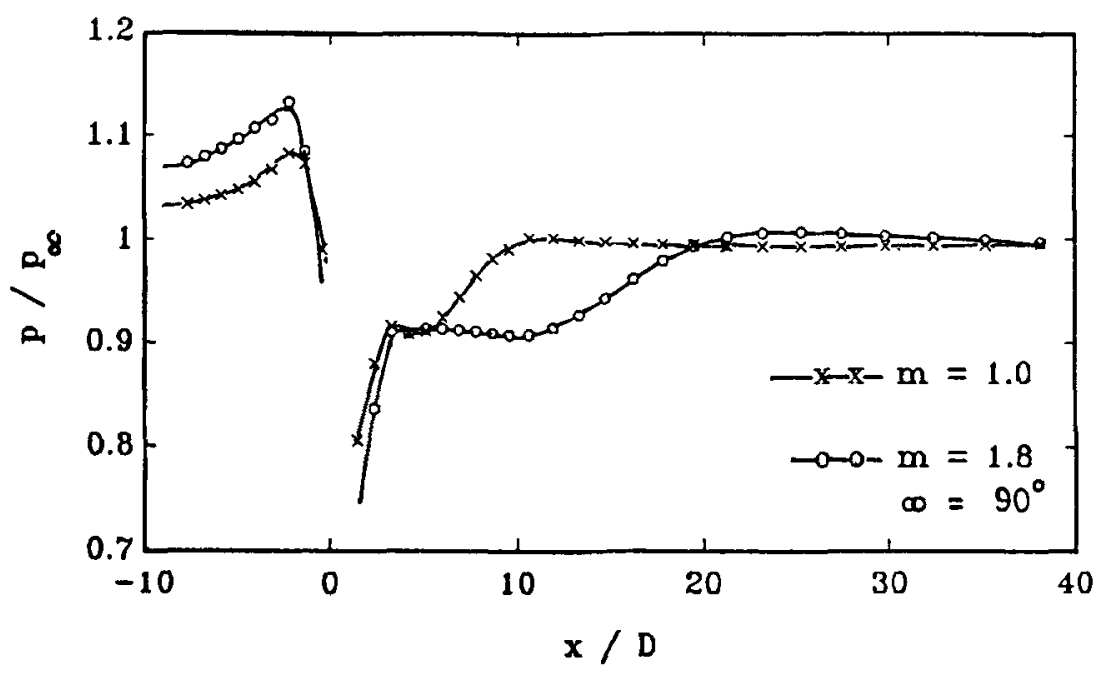

Figure 7. Computed streamwise variation of pressure ratio for different blowing rates. 


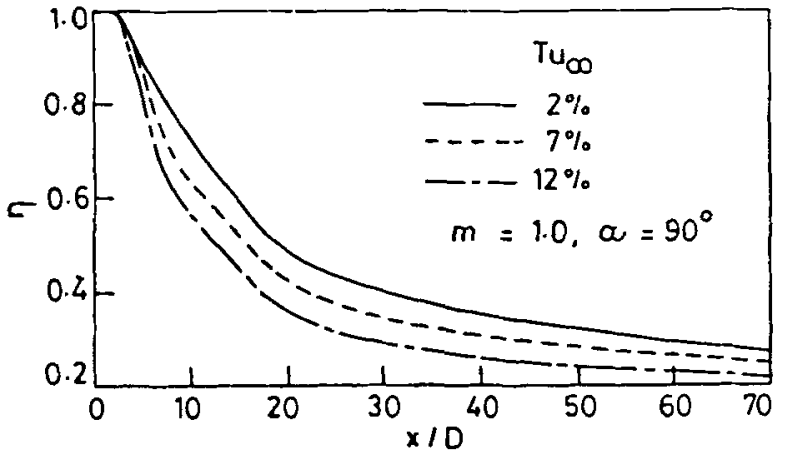

Figure 8. Streamwise variation of adiabatic film cooling effectiveness for different freestream turbulence levels.

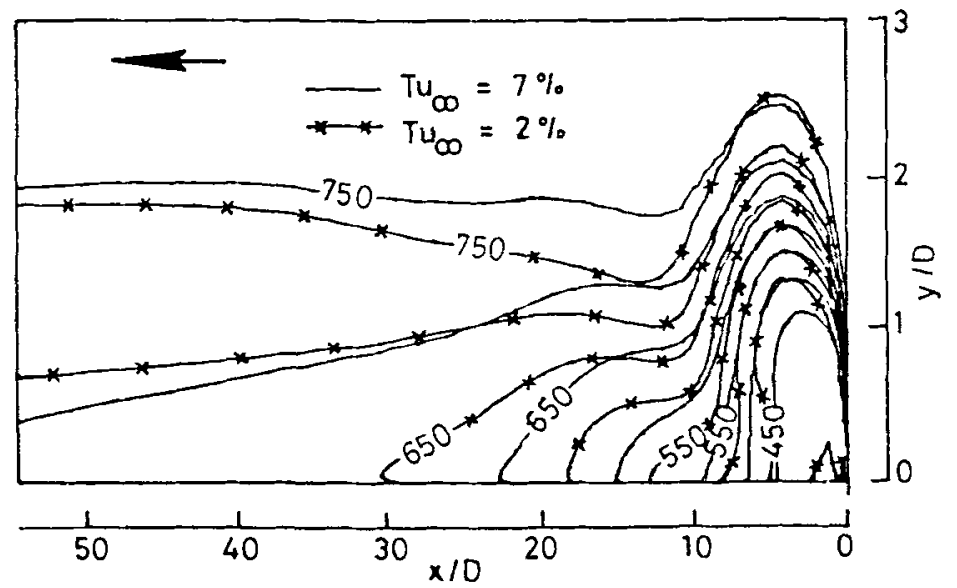

(a)

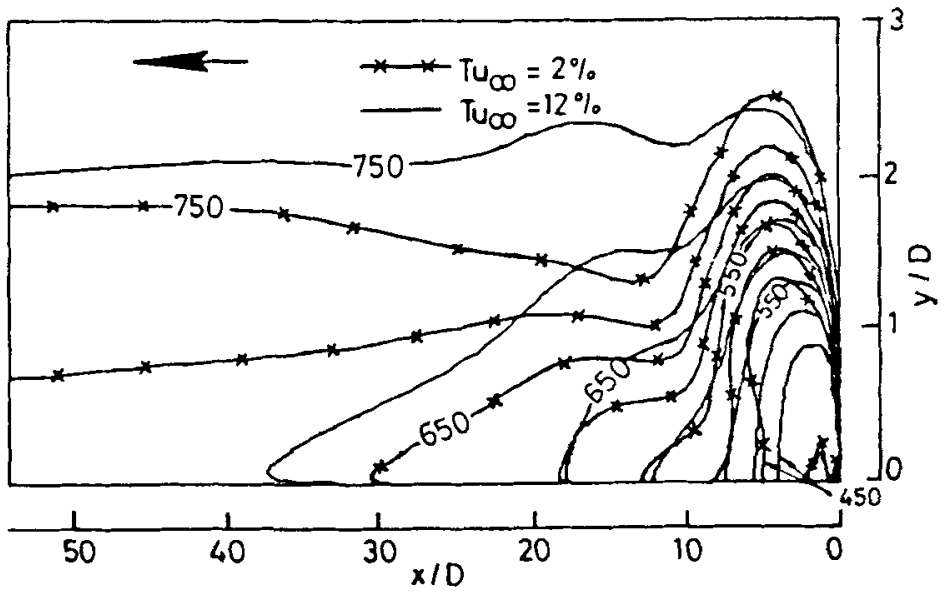

(b)

Figure 9. Computed temperature contours demonstrating effect of free-stream turbulence intensity $T u(m=1 \cdot 0, x=90)$. 
Figures $6 \mathrm{a}$ and $\mathrm{b}$ indicate cooling effectiveness for different angles of injection and blowing rates. They clearly demonstrate that the film cooling effectiveness increases in proportion to the injected mass flow rate both in the near and far field region for a low injection angle $\left(x=45^{\circ}\right)$ but only in the far field region for a high injection angle $\left(x=90^{\circ}\right)$. Increased $\alpha$ leads to a higher jet trajectory and also forms a separation bubble. The size of the separation bubble and turbulence mixing change with blowing ratios, strongly influencing near field effectiveness. Figure 5 also reveals that a shallow injection always improves cooling effectiveness because of attached coolant layer and low mixing.

The static pressure distribution along the surface is depicted in figure 7 for different blowing ratios. As seen, for $m=1$, the ratio of $p / p_{x}$ increases to a maximum of 1.08 at the upstream of jet, drops suddenly to a minimum of 0.8 at the slot trailing edge and subsequently recovers downstream. The upstream pressure rise increases with the increase of blowing ratios, indicating that the static pressure developed at the upstream corner of the slot is approximateiy equal to the injection total pressure. The flow separation downstream of the slot is pronounced with the enhancement of the blowing ratio, which is reflected by the clearly seen pressure plateau in figure 7 , especially for $m=1.8$.

6.2b Effects of free-stream turbulence: Figure 8 shows the distribution of $\eta$ as the inlet free-stream turbulence ( $T u_{x}$ ) changes for $m=1.0$ and $x=90^{\circ}$. It clearly demonstrates that as $T u_{\alpha}$ increases, $\eta$ decreases due to the pronounced mixing of the injected coolant jet and the crossflow. Effectiveness decreases almost by $25 \%$ as $T u_{\alpha}$ is increased from $2 \%$ to $12 \%$. Temperature fields, as shown in figures $9 a$ and $b$, also illustrate the change in mixing characteristics and jet trajectory as $T u_{z}$ changes. The near-field mixing is more for high $T u_{\alpha}$ resulting in a thick downstream thermal layer.
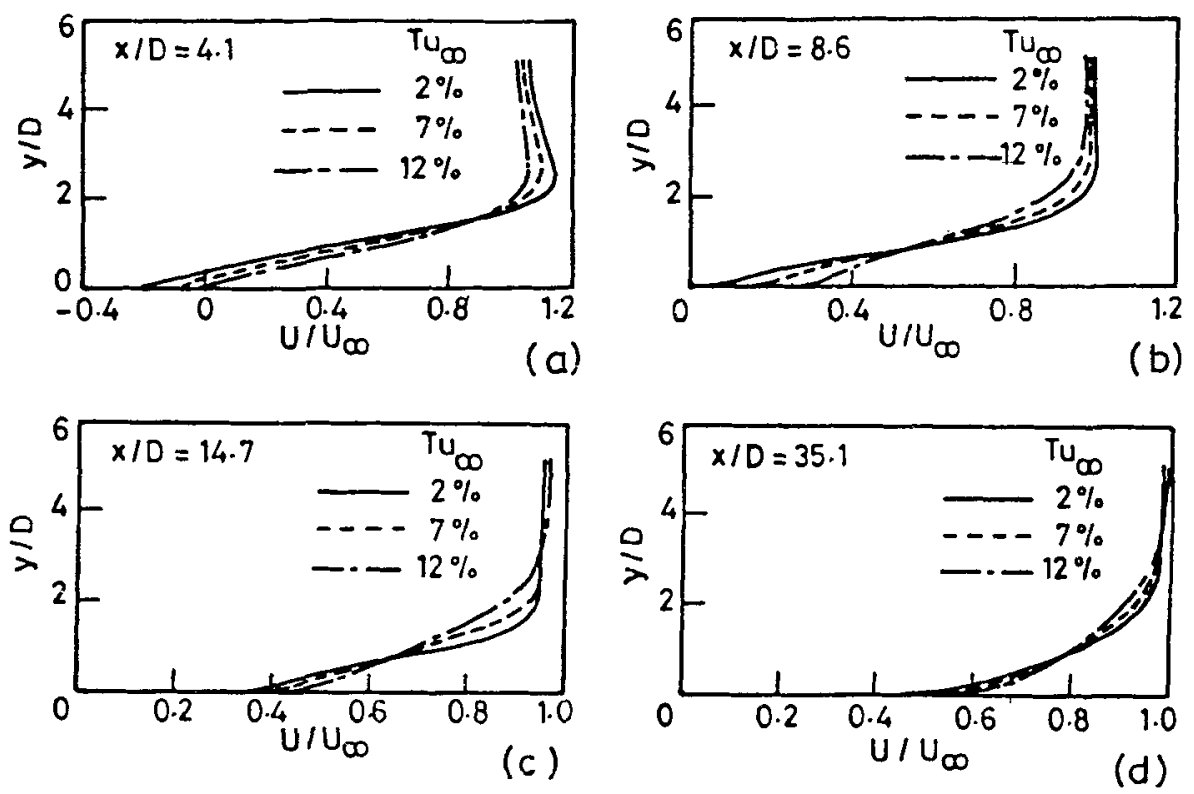

Figure 10. Effect of free-stream turbulence on mean velocity profiles $(m=1 \cdot 0$, $x=90^{\circ}$ ) at different values of $x / D$. 

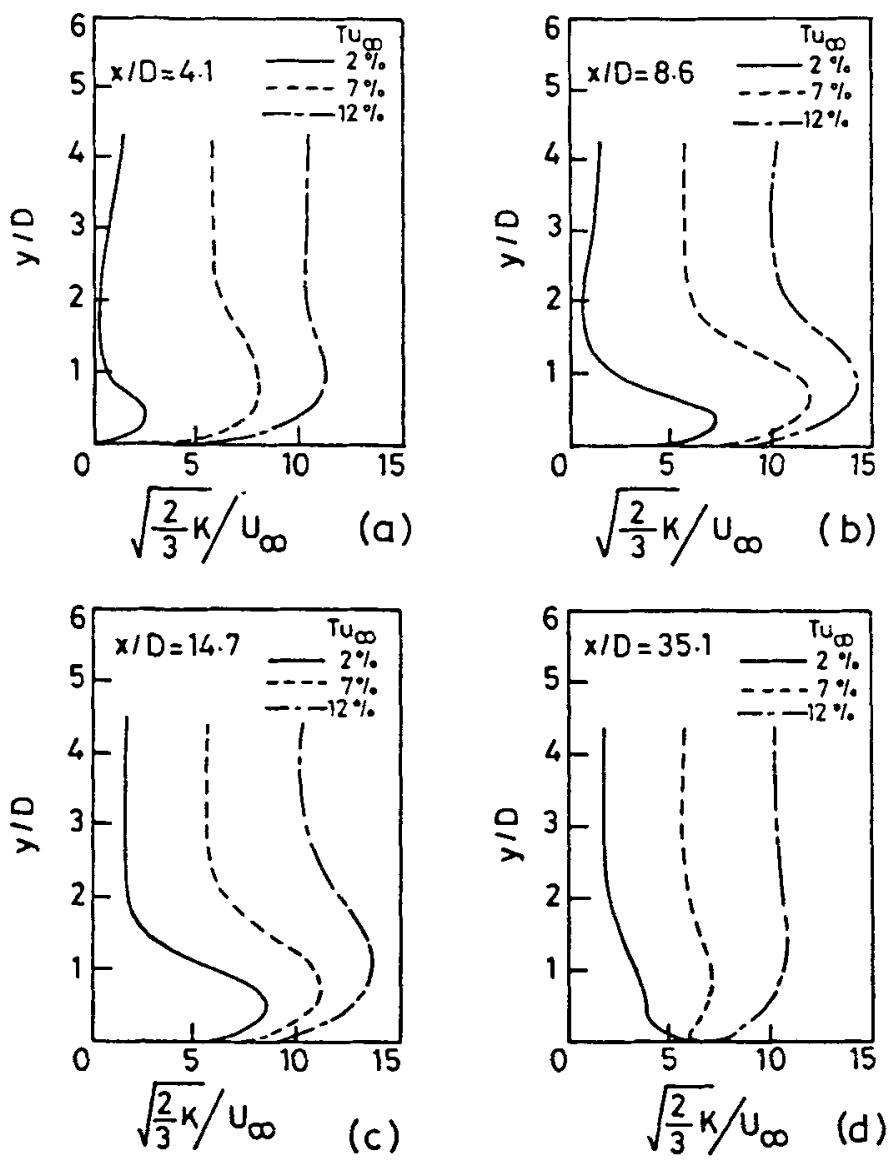

Figure 11. Effect of free-stream turbulence on turbulent kinetic energy profiles $\left(m=1 \cdot 0, \alpha=90^{\circ}\right)$ at different $x / D$ values.

The mean velocity profiles and the distribution of local turbulence levels $\left(2 / 3^{1 / 2} k / V_{\alpha}\right)$ are presented in figures 10 and 11 respectively for different spanwise locations for the same blowing rate and injection angle, $m=1 \cdot 0 ; \alpha=90^{\circ}$. Figure 10a shows a separation region and velocity overshoot for $T u_{\alpha}=2 \%$ indicating the local crossflow acceleration over the coolant jet and a corresponding drop of turbulence kinetic energy at $y / D \simeq 2$ as in figure 11a. With the enhancement of inlet turbulence intensity both the separation region and velocity overshoot decreases due to the enhanced mixing. At all other stations near the wall the gradient of velocity profiles decreases with increase of $T u_{\alpha}$. Highest turbulence occurs in the interaction zones $(x / D<20)$ due to shear layer that develops between the coolant jet and the crossflow and is seen to increase with increase of $T u_{\alpha}$. The trends of distributions of mean velocity profiles and local turbulence levels are similar to those observed by Seban (1960).

The computed normalised turbulent shear stress $\left(\widetilde{\mathrm{u}^{\prime} \mathrm{v}^{\prime}} / \mathrm{V}_{\alpha}^{2}\right)$ for $m=1.0$ and $\alpha=90^{\circ}$ are presented in figure 12 for different free-stream turbulence level. As seen, the magnitude of maximum value of the shear stress increases and its location is also shifted towards the slot as $T u_{\alpha}$ increases because of increased mixing. 


$$
m=1.0, \alpha=90^{\circ}
$$

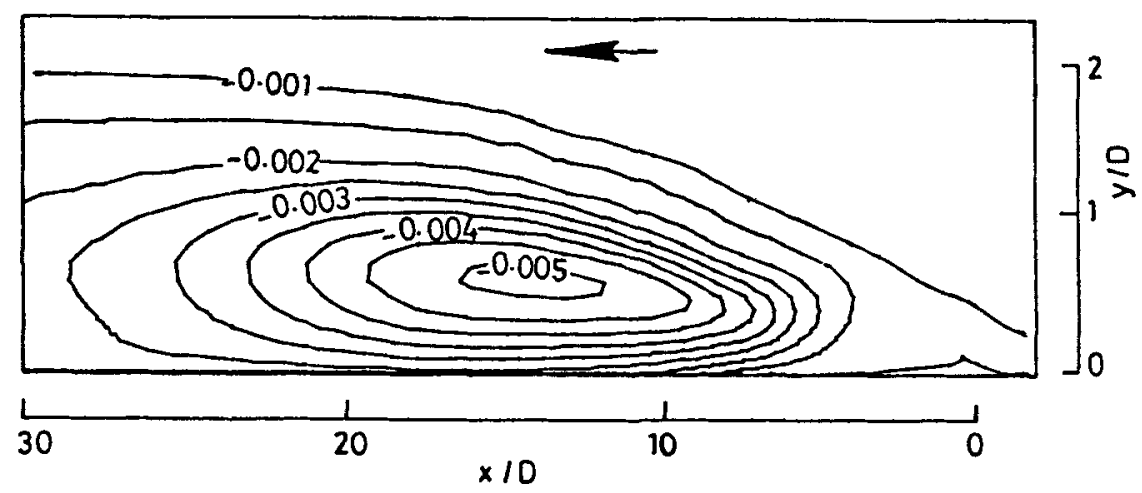

(a)

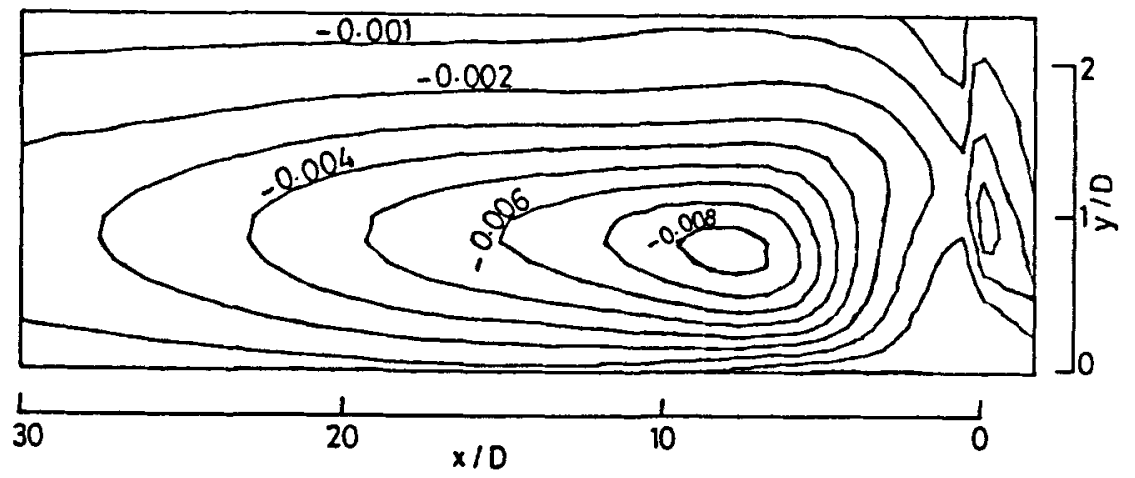

(b)

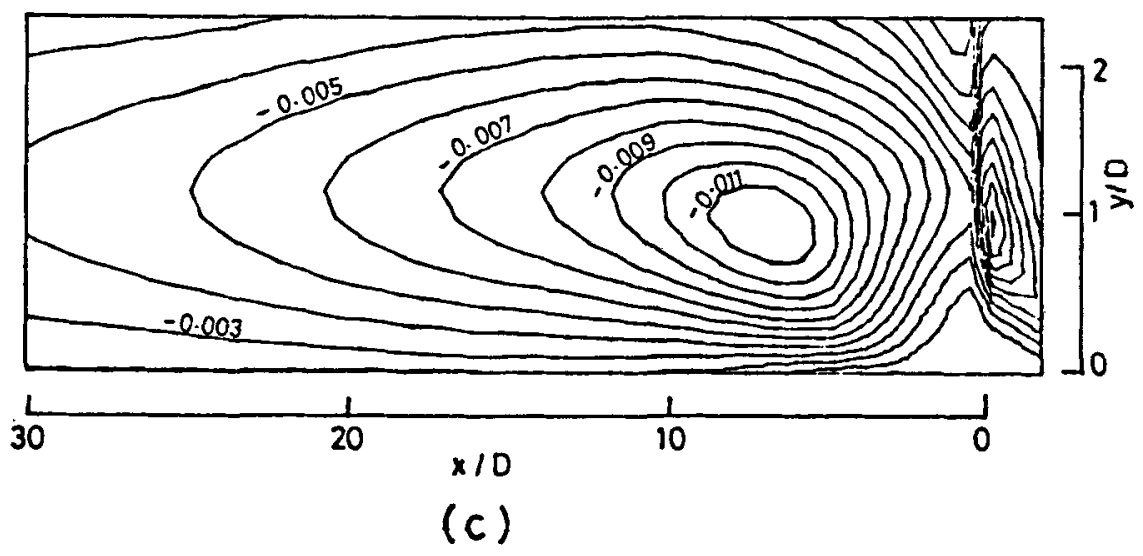

Figure 12. Predicted normalised turbulent shear stress contours for different free-stream turbulence levels $T u_{\infty}=2 \%(\mathrm{a}),=7 \%(\mathrm{~b}),=12 \%$ (c).

6.2c Turbulence quantities: The computed results presented in this sub-section aim to highlight the complex turbulent field created by the jet-crossflow interactions for changing blowing ratios and injection angles. 

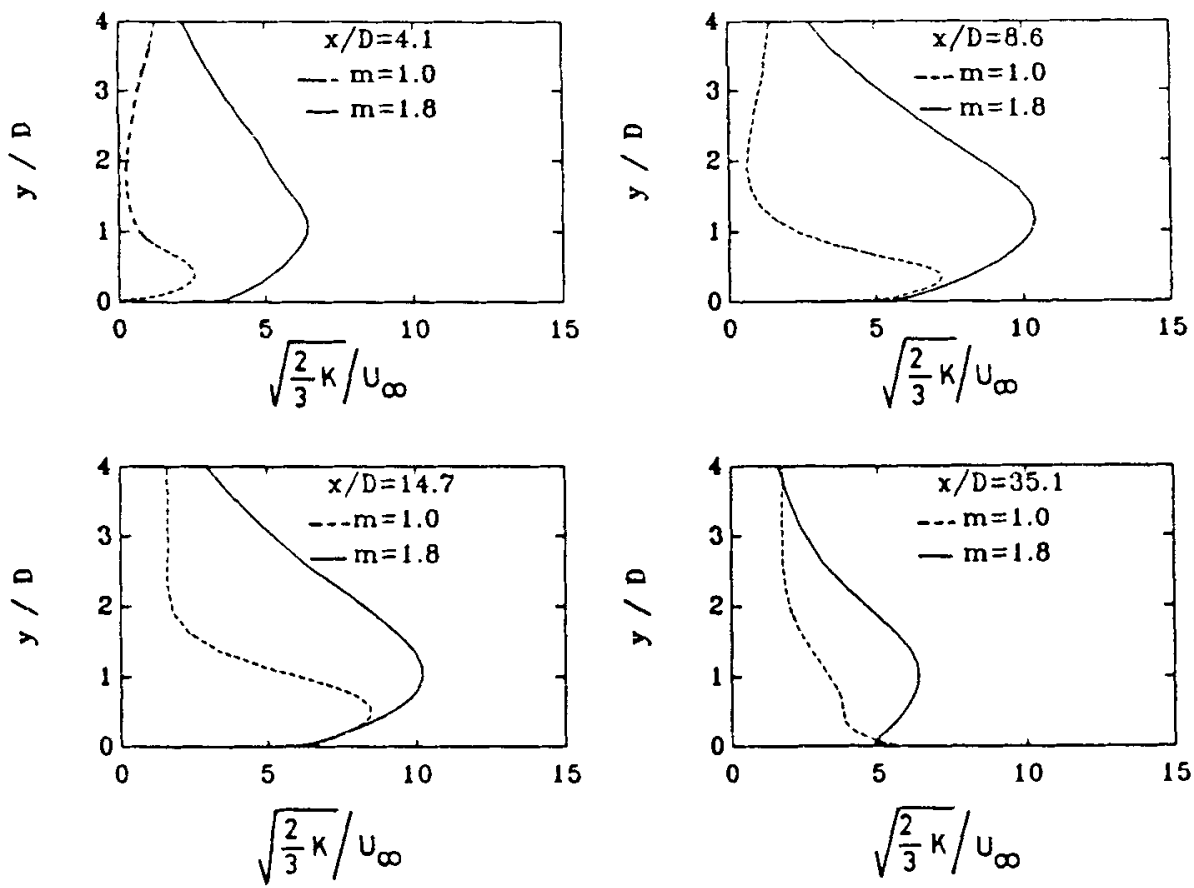

Figure 13. Predicted turbulent kinetic energy profiles at different spanwise locations and varying blowing ratios for an injection angle, $x=90$.

Figure 13 shows local turbulent kinetic energy distributions at different spanwise locations downstream of the slot exit for an angle of injection, $x=90^{\circ}$ with varying blowing ratios, $m=1.0$ and $m=1.8$. The same is represented in figure 14 for a shallow injection. $x=45^{\circ}$. The corresponding velocity profiles at the same axial locations are presented in figures 15 and 16 respectively. They clearly explain that a shallow injection produces less generation and a gradual diffusion of turbulent kinetic energy because of an attached coolant layer and a slower mixing rate. Thus, injection at low angles would result in a high film cooling effectiveness. Figure 14 also demonstrates that except very near the injection, the predicted maximum level of turbulent kinetic energy has not increased, but rather has dropped at the axial location of $x / D=14 \cdot 7$, as the blowing ratio is increased to $1 \cdot 8$. The corresponding velocity profiles (figure 16 ) reveal that for shallow injections, the momentum deficit between the injected jet and the crossfiow, which is the main source of turbulence generation for these kind of problems, has dropped with an increase of blowing ratio. But, for a high injection angle $\left(\alpha=90^{\circ}\right)$, the maximum level of turbulence is increased and the peak in turbulent kinetic energy profiles (figure 13) is shifted outwards with blowing ratios because of more jet penetration and flow mixing. Here also, the peak in turbulent kinetic energy profiles can be directly linked with the maximum momentum deficit in velocity profiles (figure 16) which demonstrate a drastic change in the relaxation behaviour of velocity and length of flow separation as blowing ratio increases. As seen in figure 14, the flow has not relaxed to turbulent boundary layer type flow even far downstream, $x / D=35$, for $m=1.8$ with $\alpha=90^{\circ}$.

As expected, because of less penetration and slow mixing of the coolant jet at a low injection angle, the maximum level of normalised shear stress has dropped to -0.0025 

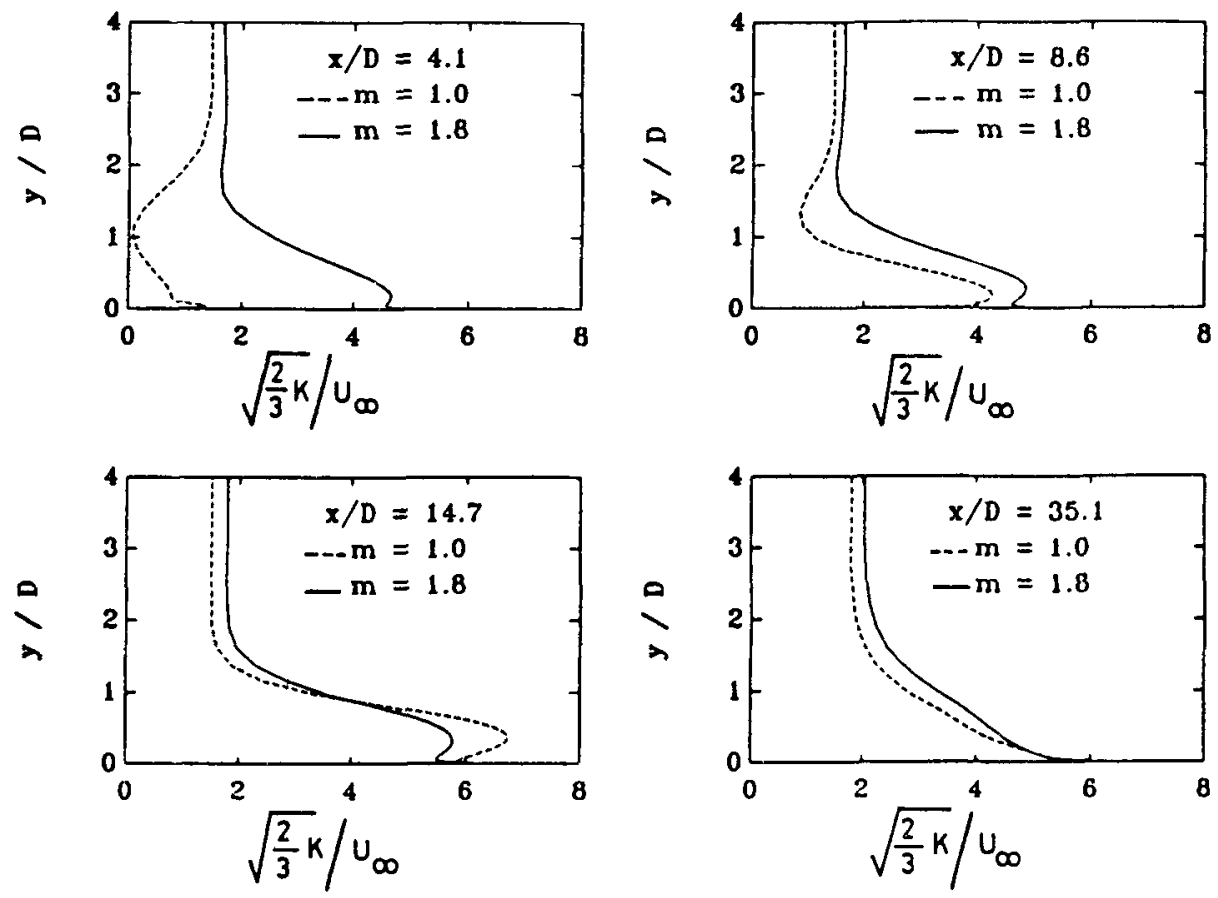

Figure 14. Predicted turbulent kinetic energy profiles at different spanwise locations for a shallow injection angle, $x=45^{\circ}$.
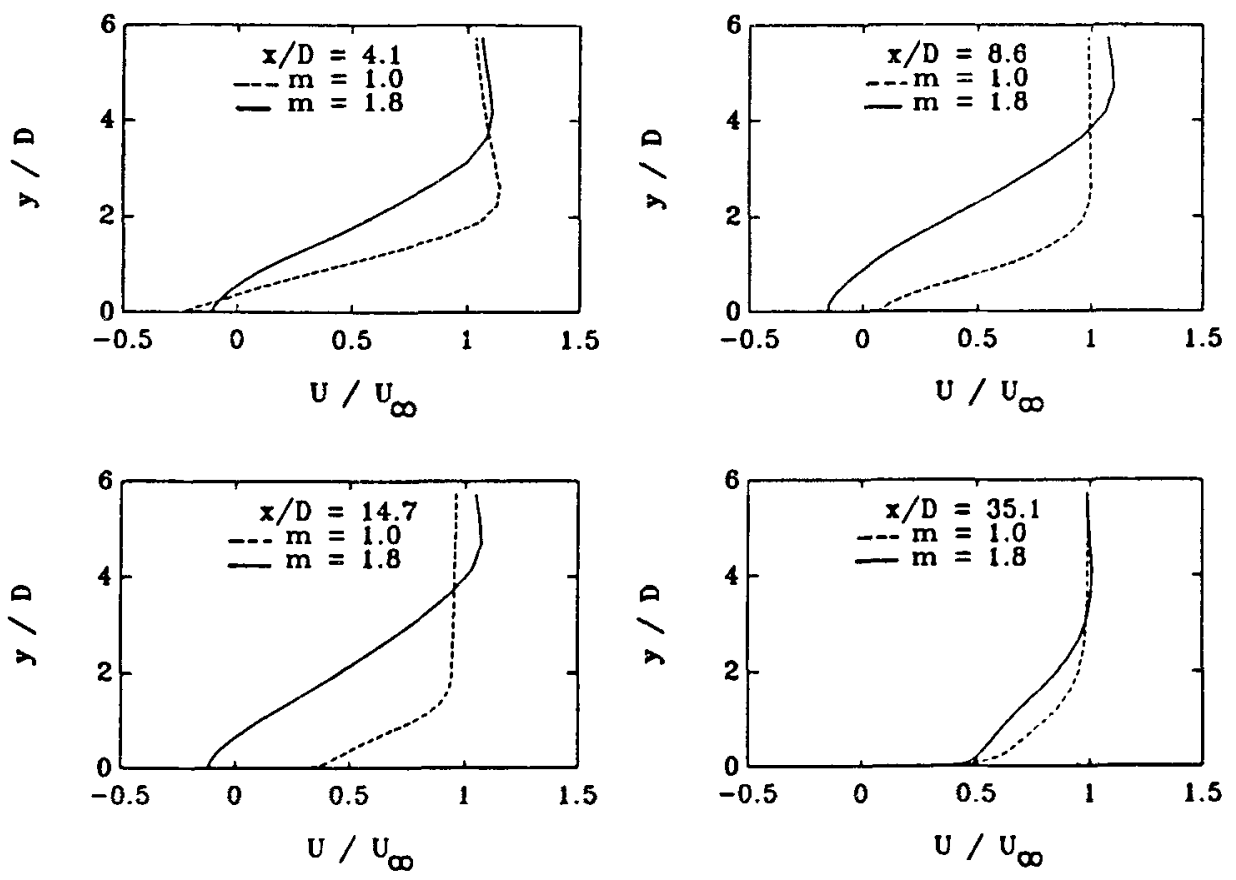

Figure 15. Predicted velocity profiles at different spanwise locations and varying blowing ratios for an injection angle, $\alpha=90^{\circ}$. 

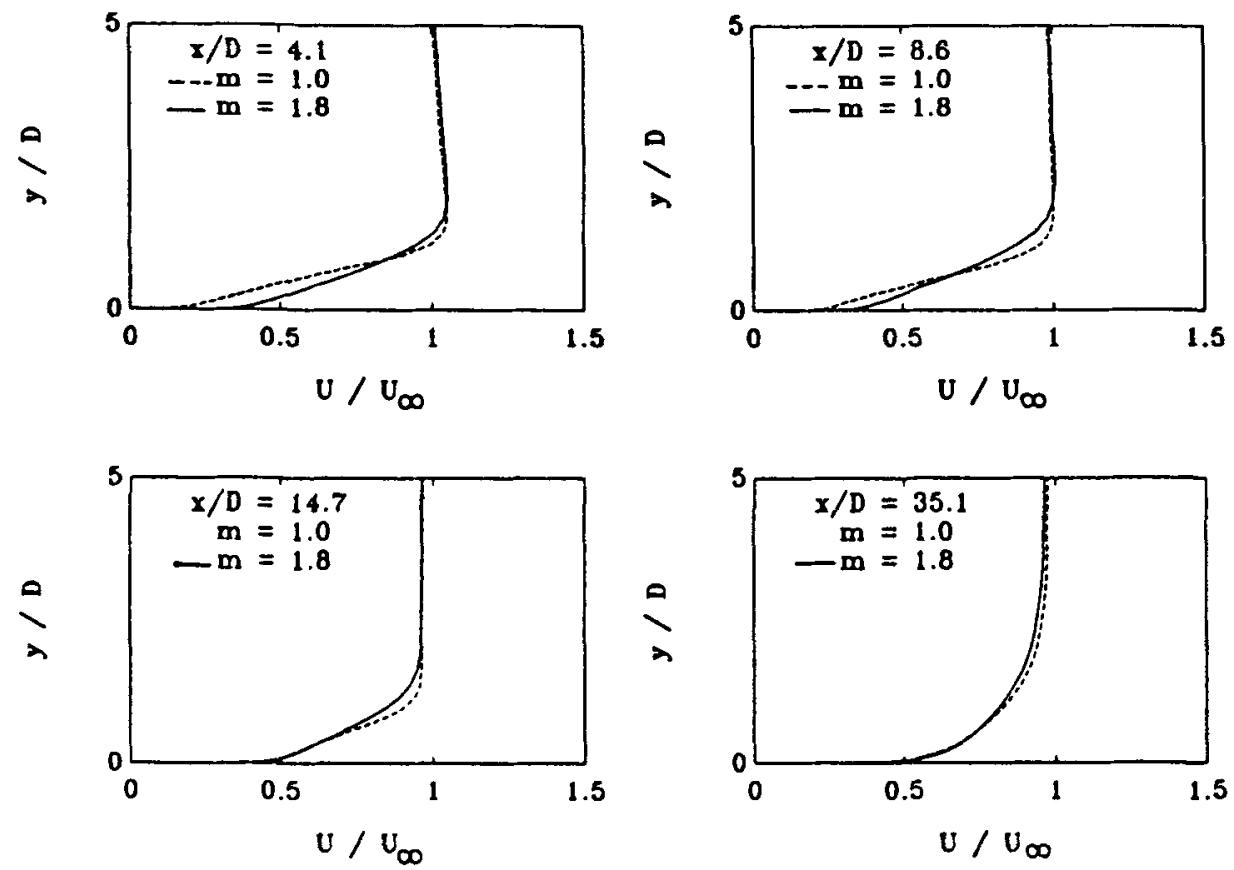

Figure 16. Predicted velocity profiles at different spanwise locations and varying blowing ratios for a shallow injection angle, $\alpha=45^{\circ}$.

from -0.005 for $m=1 \cdot 0$. For a high blowing rate $(m=1 \cdot 8)$, the maximum value of computed shear stress decreases further to -0.002 (figure 17) because of greater uniformity in velocity profiles as already explained.

\section{Conclusions}

A Navier-Stokes procedure coupled with compressible low-Reynolds number form of the $k-\varepsilon$ turbulence model in an explicit finite volume treatment is used to simulate

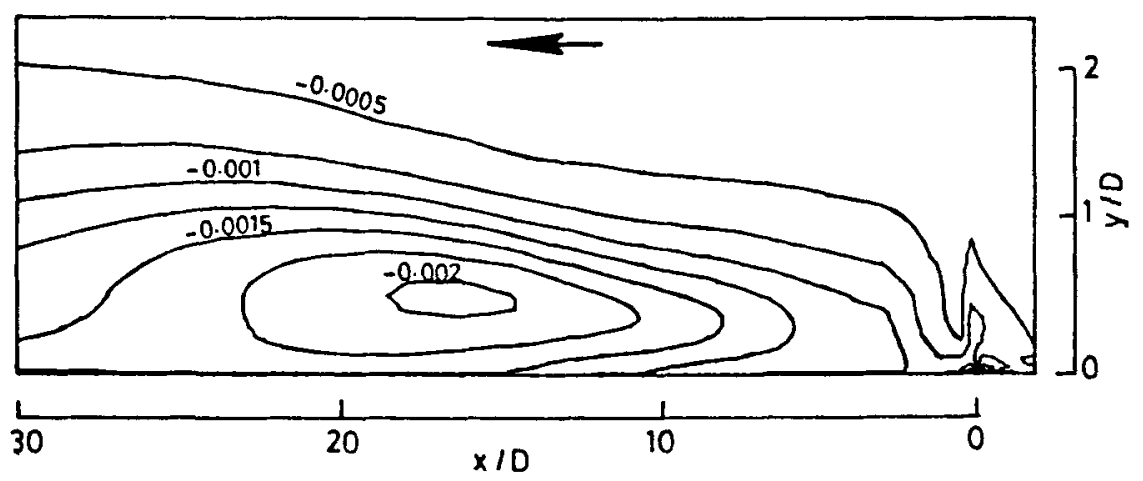

Figure 17. Computed normalised turbulent shear stress for $m=1 \cdot 8$, and $\alpha=45^{\circ}$. 
jet-crossflow interactions with changing free-stream turbulence levels, blowing ratios and injection angles for film cooling applications. The present analysis is limited to a two-dimensional simulation where free-stream conditions are similar to the gas turbine practice.

The following important points emerge from the present investigation.

(i) The injection angle and blowing ratio strongly influence the aerodynamics of a coolant jet in a crossflow and thus downstream adiabatic film cooling effectiveness. For large injection angles, the coolant penetrates more into the crossflow resulting in greater turbulence generation, followed by enhanced mixing and a rapid turbulent diffusion which leads to a faster decay of film cooling effectiveness in the near field region. A flow reversal also occurs just downstream of the slot exit. For shallow injection angles, the coolant hugs the surface with low turbulence generation and slow mixing rate, retaining its identity for a larger length. This results in high cooling effectiveness even in the far field region. For an increase in blowing rate, the overall downstream film cooling effectiveness improves due to the thickened thermal layer and more available cold fluid.

(ii) The free-stream turbulence affects the temperature distributions downstream of a coolant jet significantly by changing the mixing characteristics, local turbulence levels, mean velocity profiles and the jet trajectory. Film cooling effectiveness drops by almost $25 \%$ as inlet free-stream turbulence increases from 2 to $12 \%$.

(iii) Shallow injection changes the computed turbulent field indicating less generation and a gradual diffusion of turbulent kinetic energy. At a relatively low injection angle, an increase of blowing rate reduces the momentum deficit and in turn the maximum turbulence level which confirms that the shear layer between the crossflow and the coolant jet is the main source of turbulence generation for this kind of problem.

\section{List of symbols}

$\begin{array}{ll}D & \text { coolant injection slot width; } \\ D R & \text { density ratio }=\rho_{s} / \rho_{x} ; \\ e & \text { total energy per unit mass; } \\ \mathbf{F}, \mathbf{G} & \text { flux vectors; } \\ l & \text { turbulence length scale; } \\ \mathbf{M} & \text { Mach number; } \\ m & \text { blowing ratio }=(\rho V)_{s} /(\rho V)_{x} ; \\ m_{s} & \text { coolant mass flow rate; } \\ \mathrm{Pr}, \mathrm{Pr}_{t} & \text { Prandtl numbers; } \\ p & \text { pressure; } \\ q_{i} & \text { Cartesian components of heat flux vector; } \\ R & \text { gas constant; } \\ \operatorname{Re} & \text { Reynolds number; } \\ \bar{S} & \text { surface normal to a side of the cell; } \\ T & \text { temperature; } \\ T_{a w} & \text { adiabatic wall temperature; } \\ T_{r} & \text { mainstream recovery temperature; } \\ T_{r s} & \text { recovery temperature of coolant; } \\ \Delta t & \text { time step; }\end{array}$


Tu turbulence intensity

U solution vector;

$u_{\text {: }} \quad$ friction speed $=\left(\tau_{w} / \rho\right)^{12}$;

u. $\iota$ Cartesian velocity components;

$\checkmark$ magnitude of velocity vector;

$x, y \quad$ Cartesian coordinates (defined in figure 1);

$y^{+} \quad$ law-of-the-wall coordinate $=y\left[\left(\rho^{\prime} \mu\right)\left(\delta u, \delta y^{\prime}\right)\right]_{w^{\prime}}{ }^{2}$;

$\alpha \quad$ injection angle of the coolant jet:

$\delta_{i j} \quad$ Kronecker delta;

$\eta \quad$ adiabatic film effectiveness $=\left(T_{a x}-T_{r}\right) /\left(T_{r s}-T_{r}\right)$;

$\mu, \mu_{t} \quad$ molecular and turbulent viscosities;

$\rho$ density;

$\tau_{i j} \quad$ Cartesian components of stress tensor;

$\Omega \quad$ cell volume.

\section{Superscripts}

fluctuating quantity in density-weighted time averaging;

- density-weighted time-averaged quantity.

\section{Subscripts}

i.j logical cell indices in space;

$s \quad$ coolant slot exit conditions:

$t$ turbulent:

w wall condition;

$x \quad$ mainstream conditions at the inlet plane.

\section{References}

Andreopoulos J, Rodi W 1984 Experimental investigation of jets in a crossflow. J. Fluid Mech. 138: $93-127$

Brown A. Minty A G 1975 The effects of mainstream turbulence intensity and pressure gradient on film cooling effectiveness for cold air injection slits of various ratios. ASME Paper No. 75-WA/HT-17

Cebeci T. Smith A M O 1974 Analysis of turbulent boundary layers (New York. San Francisco. London:Academic Press)

Favre A 1965 Equations des Gaz Turbulents Compressible: I. Formes Generales. J. Mec. 4: $361-390$

Foster N W, Lampard D 1980 The flow and film cooling effectiveness following injection through a row of holes. ASME. J. Eng. Power 102: 584-588

Goldstein R J 197! Film cooling. Adrances in heat transfer (New York and London: Academic Press) vol. 7. pp. 321-379

Jameson A. Schmidt W. Turkel E 1981 Numerical solutions of the Euler equations by finite volume methods using Runge-Kutta time-stepping schemes. AIAA Paper 81-1259

Jumper G W. Elrod W C. Rivir R B 1991 Film cooling effectiveness in high-turbulence flow. ASME, J. Turhomach. 113:479-483

Ko S. Liu D 1980 Experimental investigations of effectiveness, heat transfer coefficient and turbulence of film cooling. AIAA J. 18: 907-913 
Kadotani K, Goldstein R J 1979 On the nature of jets entering a turbulent flow, Part A - Jet-mainstream interaction. J. Eng. Power 101: 459-465

Kunz R, Lakshminarayana B 1991 Stability of explicit 3-D Navier-Stokes/ $k-\varepsilon$ procedures in complex internal flow calculations. AIAA Paper 9I-0018, pp. 1-14

Lam C K G, Bremhorst K 1981 A modified form of the $k-\varepsilon$ model for predicting wall turbulence. ASME J. Fluids Eng. 103: 456-460

MacCormack R W, Paullay A J 1972 Computational efficiency achieved by time splitting of finite difference operators. AIAA Paper 72-154

Papell S S 1960 Effect on gaseous film cooling injection through angled slots and normal holes. NASA TN-D-299

Petersen D R Eckert E R G, Goldstein R J 1977 Film cooling with large density differences between the mainstream and secondary fluid measured by heat-mass transfer analogy. ASME, J. Heat Transfer 99: 620-627

Pietrzyk J R, Bogard D G, Crawford M E 1989 Hydrodynamics measurements of jet in crossflow for gas turbine film cooling applications. ASME, J. Turbomach. 111: 139-145

Seban R A 1960 Effects of initial boundary-layer thickness on a tangential injection system. ASME J. Heat Transfer 82: 392-393

Sinha A K, Bogard D G, Crawford M E 1990 Film cooling effectiveness of a single row of holes with variable density ratio. ASME Paper No. 90-GT-43 